\title{
Galisonian logic devices and data availability: revitalising Upper Palaeolithic cultural taxonomies Ben Marwick*
}

* Department of Anthropology, University of Washington, 314 Denny Hall, Box 353100, Seattle, WA 98195-3100 USA

This paper was published as:

Marwick, B. (2019). Galisonian logic devices and data availability: Revitalising Upper Palaeolithic cultural taxonomies. Antiquity, 93(371), 1365-1367. https://doi.org/10.15184/aqy.2019.131

as a response to the debate lead article:

Reynolds, N. \& Riede, F.. 2019. House of cards: cultural taxonomy and the study of the European Upper Palaeolithic. Antiquity 93: 1350-58. https://doi.org/10.15184/aqy.2019.49

Reynolds and Riede's (2019) call for caution in the use of cultural taxonomies in the study of stone artefact assemblages is welcome. In my contribution to this debate, I draw on work in the philosophy of science to explore the implications for archaeology should researchers adopt Reynolds and Riede's proposals - particularly those concerning data types and data availability.

The study of cultural taxonomies defined by lithic materials currently resembles a Lakatosian degenerative research programme. Lakatos (1978) proposed that a scientific research programme can be recognised by a 'hard core' of established central tenets that are the assumptions upon which all work is based, and a 'protective belt' of predictions and hypotheses that are actively under investigation. At any point in time, a programme is progressive, stagnant or degenerating. Reynolds and Riede note that cultural taxonomies rarely engage with work on archaeological systematics and epistemology, which should represent the hard core of a taxonomic programme. Without such an emphasis, the cultural taxonomy programme-as 
currently constituted in stone artefact archaeology — is stagnant, or even degenerating. What steps can we take to build a substantial hard core for this programme? Unfortunately, Reynolds and Riede are silent on the question of which of the competing approaches (e.g. essentialist typological thinking or evolutionist population thinking) will be most productive for Palaeolithic archaeology. The answer may only become apparent after large-scale comparative work becomes possible. With data availability of archaeological journal articles currently at around 50 per cent of published papers (Marwick \& Pilaar Birch 2018), we are, however, still a decade or two away from the routine meta-analysis of data from multiple archaeological publications, such as is routine in fields such as psychology and oncology.

If archaeologists heed Reynolds and Riede's call for large-scale comparative work, they may stimulate a major shift in what counts as data, and how it is collected and shared. We can see a description of these types of shifts in Galison's (1997) analysis of experimental particle physics. In his book Image and Logic, he describes a historical sequence of two incompatible research traditions in particle physics, with the transition between the two driven predominantly by technological changes. The earlier tradition represents particles through the hand-collection of photographic images. The time-consuming nature of generating such images meant that visual methods were best suited for the detailed study of a single image, and often a single event served as evidence for a novel claim. This was succeeded by a tradition similar to contemporary practices that uses electrical sensors and statistical methods to collect masses of quantitative data. Galison calls this the 'logic tradition', which allows researchers to collect data rapidly_albeit in less detail than individual photographs - from a large number of events. The accumulation of large volumes of sensor data has made it possible for physicists to conduct statistical analysis in order to evaluate claims.

We can find close parallels in the practice of stone artefact archaeology to the traditions described by Galison for microphysics. As Reynolds and Riede note, hand-drawn or photographic illustrations of a small number of visually distinctive stone artefacts are often presented as the primary evidence in support of cultural taxonomic claims and interpretations. We also have a parallel, overlapping tradition that adds quantitative metric and attribute data for all individual pieces in an assemblage. Currently, for most researchers, these data are collected 
by hand with callipers and similar hand-operated instruments. Callipers resemble a Galisonian 'logic device' in their more rapid collection of less detailed data-relative to an illustration - from a stone artefact. This is desirable, as it produces data that are more representative of past behaviours than a small set of artefacts subjectively selected for illustration. Callipers are therefore vital for generating the full datasets that Reynolds and Riede argue to be urgently needed to conduct meaningful, robust cultural taxonomic research. To share these detailed assemblage data, we can directly borrow repositories, licences and policies from other research communities, in which these norms have been established for many years. Data availability is already an established norm in fields such as palaeoproteomics that produce masses of quantifiable data. Within the social sciences, the American Journal of Political Science, for example, only publishes papers after a reviewer has verified that the data and analysis code submitted along with the manuscript can reproduce the specific results presented. Imagine how efficiently large-scale comparative work in Palaeolithic archaeology could be achieved, if we required similar quality control for our journals, in order to ensure that detailed datasets were readily available.

Although callipers and related instruments are an important part of the toolkit for improving cultural taxonomies, a more striking analogy to the Galisonian logic device is the application of computer vision (e.g. the OpenCV library, Bradski 2000) and geometric morphometric statistics to images of artefacts (either two-or three-dimensional photogrammetric models). This tradition, which is emerging in the archaeological literature (e.g. Buchanan et al. 2014; Reide et al 2019), allows us to both see the artefact, and to extract their curves, outlines and surfaces rapidly, using automatic computer vision algorithms. This gives us a much richer dataset than linear calliper measurements, and thus a much broader scope for statistical comparison. Furthermore, if these methods are implemented in an open source programming language, such as R or Python, we would have a logical trace from each artefact's image to its statistical representation. This is important for responding to Reynolds and Riede's observation that making data available is only a hollow victory, unless we communicate descriptions of how such data are produced. While glossaries are part of the solution to improving data re-usability, a thorough understanding of the logic and usefulness of data is only possible if scripts are available 
that document the transformation of data as they move from collection to published summary tables or visualisations.

Improved data availability and methodological transparency from archaeologists working on stone artefacts will do more than improve the robustness of cultural taxonomic work. It will also help to close the gap between the practice of Palaeolithic archaeology and the ideals of archaeological science, specifically, the gap around the first Mertonian ideal of communality (Mulkay 1976). Communality is the idea that scientists value common ownership of scientific results and methods, and share both freely. A closer adherence to this principle will have the additional benefit of helping to decolonise archaeology. We often work on cultures to which we do not belong, and we take away their remains and sometimes never return them, putting them into museum or university research collections. These practices may be at odds with the stewardship responsibilities of the indigenous and local groups with whom we work, and may have analogies to the economic and political activities of Western colonising nations (Atalay 2006). Making available the data extracted from these specimens is a necessary process in decolonising archaeology and supporting participation from our indigenous, community and under-resourced collaborators. Data availability is important not only for improving our cultural taxonomies, but also for an ethical and socially just practice of archaeological research.

\section{Acknowledgements}

Thanks to Gayoung Park for reading an earlier draft and offering suggestions for improvements.

\section{References}

Atalay, S. (2006). Indigenous archaeology as decolonizing practice. American Indian Quarterly, $30(3 \& 4) 280-310$.

Bradski, G., 2000. The OpenCV Library. Dr. Dobb's Journal of Software Tools. Available at http://www.drdobbs.com/open-source/the-opencv-library/184404319 (accessed 5 August 2019).

Buchanan, B., O’Brien, M.J., Collard, M., 2014. Continent-wide or region-specific? A geometric morphometrics-based assessment of variation in Clovis point shape. Archaeological and Anthropological Sciences 6, 145-162. 
GaLison, P. 1997. Image and logic: a material culture of microphysics. Chicago (IL): University of Chicago Press.

Lakatos, I. 1978. The methodology of scientific research programmes. New York: Cambridge University Press.

Marwick, B. \& S.E. PilaAr Birch. 2018. A standard for the scholarly citation of archaeological data as an incentive to data sharing. Advances in Archaeological Practice 6: 125-43.

Mulkay, M. 1976. Norms and ideology in science. Social Science Information 15: 637-56.

Riede, F., Hoggard, C., Shennan, S., 2019. Reconciling material cultures in archaeology with genetic data requires robust cultural evolutionary taxonomies. Palgrave Communications 5, 1-9. Reynolds, N. \& F. Riede. 2019. House of cards: cultural taxonomy and the study of the European Upper Palaeolithic. Antiquity XX: XX 